\title{
Fault warning method based on extreme learning regression and fuzzy reasoning
}

\author{
LI Gang ${ }^{1}$, QIU Chen-guang ${ }^{1}$, CAO Shuai ${ }^{1}$, WANG Ya-ou ${ }^{* 2}$ \\ State Grid Jiangsu Electric Power Co., Ltd ${ }^{1}$ \\ Nanjing 210024 China; Jiangsu Fangtian Co., Ltd ${ }^{2}$, Nanjing 210036 China
}

\begin{abstract}
Aiming at the problem of condition monitoring of thermal power units, a fault early warning method based on fuzzy learning machine is proposed. The extreme learning regression model between monitoring parameters is established by using real-time data. Then the estimated value is fuzzed and used for fuzzy reasoning, finally, the fault diagnosis results of the unit under small abnormal state are obtained. The simulation data of a $1000 \mathrm{MW}$ unit is used for verification test and results show that the proposed method is reliable and accurate which is suitable for thermal unit state warning.
\end{abstract}

\section{INTRODUCTION}

In recent years, with the deepening of the reform of the power market, the demand of power grid for peak load regulation capacity of thermal power units is increasing, so the uncertainty of unit operation rises which leads to the increase of failure probability to a certain extent. According to statistics, the number of unplanned outage of $1000 \mathrm{MW}$ units in Jiangxi power grid in 2019 is about 1, number of $600 \mathrm{MW}$ and $300 \mathrm{MW}$ units are also greater than $0.6^{[1]}$, which caused huge economic losses and adverse effects. At present, when the unit DCS alarm emerges, equipment failure has resulted in serious deterioration, which can only be handled by decreasing the load and applying for emergency shutdown in site, causing great loss to power generator enterprises. Therefore, in the early stage of fault, the use of technical methods for the warning of the tiny deterioration in thermal unit is particularly important.

The operation data of generators is the only source to obtain the working state of the unit. Mining the hidden state information in the data deeply provides a feasible and effective method for the unit state warning technology research. Zhang Z Y applied data mining to early warning of actual power station ${ }^{[2]}, \mathrm{Fu} \mathrm{W}$ A studied the fault early warning model of extreme random tree algorithm ${ }^{[3]}$; Han W L applied PCA method and MSET method to unit state early warning ${ }^{[4]}$. In addition, there are other studies on early warning methods such as correlation analysis, neural network, and so on ${ }^{[5-6]}$.

Compared with other data modeling methods, the extreme learning machine has unique advantages in the reliability and accuracy of the model ${ }^{[7]}$. In this paper, the extreme learning machine is introduced to establish the monitoring and warning model of the thermal power unit parameters, and the specific fault state is determined by using the fuzzy inference based on fault library. Based on a $1000 \mathrm{MW}$ thermal power simulation system, the boiler fault is simulated to verify the proposed method.

\section{Fault early warning methods}

\subsection{Extreme learning regression}

ELM (Extreme Learning Machines) is actually a forward propagation neural network with a single hidden layer, in which neurons can use common activation functions, such as sigmoidal function and radial basis function, etc. compared with the traditional neural network, ELM converges faster and can avoid falling into local optimum. This section briefly introduces the principle of its algorithm.

For the AAELM, the input and output signals are the same parameters, namely $\boldsymbol{x}_{j}=\boldsymbol{t}_{j}=\boldsymbol{X}_{T}(j), j=1, \mathrm{~K}, N_{T}$. Given the training sample set $\boldsymbol{X}_{T}$, the excitation output function of the hidden layer node is $G\left(\boldsymbol{a}_{i}, b_{i}, \boldsymbol{X}_{T}\right)$. The number of nodes in the hidden layer is chosen as $L$, activation function is chosen as sigmoidal function. The process of AAELM algorithm is as follows:

(1) Randomly generate parameter values of node functions in hidden layer $\left(\boldsymbol{a}_{i}, b_{i}\right), i=1, \mathrm{~K}, L$;

(2) Calculation the output matrix of hidden layer $H$;

$$
H=\left[\begin{array}{c}
\boldsymbol{h}\left(\boldsymbol{x}_{1}\right) \\
\mathrm{M} \\
\boldsymbol{h}\left(\boldsymbol{x}_{N}\right)
\end{array}\right]=\left[\begin{array}{ccc}
G\left(\boldsymbol{a}_{1}, b_{1}, \boldsymbol{x}_{1}\right) \mathrm{L} & G\left(\boldsymbol{a}_{L}, b_{L}, \boldsymbol{x}_{1}\right) \\
\mathrm{M} & \mathrm{O} & \mathrm{M} \\
G\left(\boldsymbol{a}_{1}, b_{1}, \boldsymbol{x}_{N}\right) \mathrm{L} & G\left(\boldsymbol{a}_{L}, b_{L}, \boldsymbol{x}_{N}\right)
\end{array}\right]_{N \times L}
$$

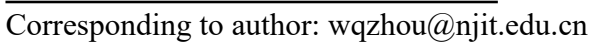




$$
\hat{\beta}=D T
$$

If $H^{T} H$ is not a singular matrix, then:

$$
D=\left(H^{T} H\right)^{-1} H^{T}
$$

If $H H^{T}$ is not a singular matrix, then:

$$
D=H^{T}\left(H H^{T}\right)^{-1}
$$

(4) calculate the regression value of parameters

$$
\boldsymbol{X}_{R}=\boldsymbol{H} \hat{\beta}
$$

\subsection{Fuzzy inference}

The fault identification is actually based on the distance comparison of various models, which judges and recognizes the current fault state according to certain rules. The common distance function is:

$$
d_{j}\left(u_{0}, u_{j}\right)=\sqrt{\sum_{i=1}^{n}\left(z_{i}-z_{i j}\right)^{2}}
$$

Where $d_{j}\left(u_{0}, u_{j}\right)$ is the distance between the fault $u_{0}$ to be identified and the typical fault mode $u_{j}$. It is obvious that the smaller value means the greater possibility of such failure. $z_{j}$ is the fault symptom value of the $i^{\text {th }}$ symptom parameter, and $z_{i j}$ is the symptom value of the $i^{\text {th }}$ symptom parameter under the $j^{\text {th }}$ typical fault which are obtained by fuzzifying function. The membership function is as follow:

$$
\begin{gathered}
\mu_{u_{j}}\left(u_{0}\right)=1-\frac{d_{j}\left(u_{0}, u_{j}\right)}{D} \\
D=\max \left(d_{j}\left(u_{0}, u_{j}\right)\right)
\end{gathered}
$$

Obviously, as shown in formula (7), the closer to 1 the degree of membership is, the more possibility for this type of failure to occur.

\section{Equipment fault identification}

\subsection{Expert knowledge base of boiler leakage}

Fault knowledge base is the basis of fault identification, it describes the relationship of patterns between fault categories and symptom parameters of various fault. Take boiler leakage as an example, this paper proposes the monitoring parameters and fault modes shown in table 1 and table 2 .

Tab.1 Four Tubes Leak symptom parameter set

\begin{tabular}{ll}
\hline Variate & Description of symptom parameters \\
\hline$x_{1}$ & Main steam pressure \\
$x_{2}$ & Reheat steam pressure \\
$x_{3}$ & Smoke temperature deviation on both sides of the water wall \\
$x_{4}$ & Flue gas temperature deviation on both sides of economizer \\
$x_{5}$ & Flue temperature deviation on both sides of the superheater \\
$x_{6}$ & Flue temperature deviation on both sides of reheater \\
$x_{7}$ & Induced draft fan stator blade opening degree \\
$x_{8}$ & Cooling water flow \\
\hline
\end{tabular}

Tab.2 Four Tubes Leak expert knowledge base

\begin{tabular}{ccccc}
\hline Variate & Water wall $u_{1}$ & economizer $u_{2}$ & Superheater $u_{3}$ & Reheater $u_{4}$ \\
\hline$x_{1}$ & -0.5 & -0.5 & -0.5 & 0 \\
$x_{2}$ & 0 & 0 & 0 & -0.5 \\
$x_{3}$ & 1 & 0.5 & 0 & 0 \\
$x_{4}$ & 0 & 1 & 0 & 0 \\
$x_{5}$ & 0 & 0 & 1 & 0 \\
$x_{6}$ & 0 & 0 & 0 & 1 \\
$x_{7}$ & 0.5 & 0.5 & 1 & 0.5 \\
$x_{8}$ & 0.5 & 0.5 & 1 & 0 \\
\hline
\end{tabular}

\subsection{Unit simulation}

The simulation system of a $1000 \mathrm{MW}$ thermal power unit is used to simulate various operation states to verify the effectiveness of the method proposed in this paper for early diagnosis of boiler leakage faults.
The simulation system was used to set different types of boiler leakage faults, and the change curves of various typical symptom parameters are obtained. Figure 1 shows the variation trend of parameters under the eco leakage fault on side A. 

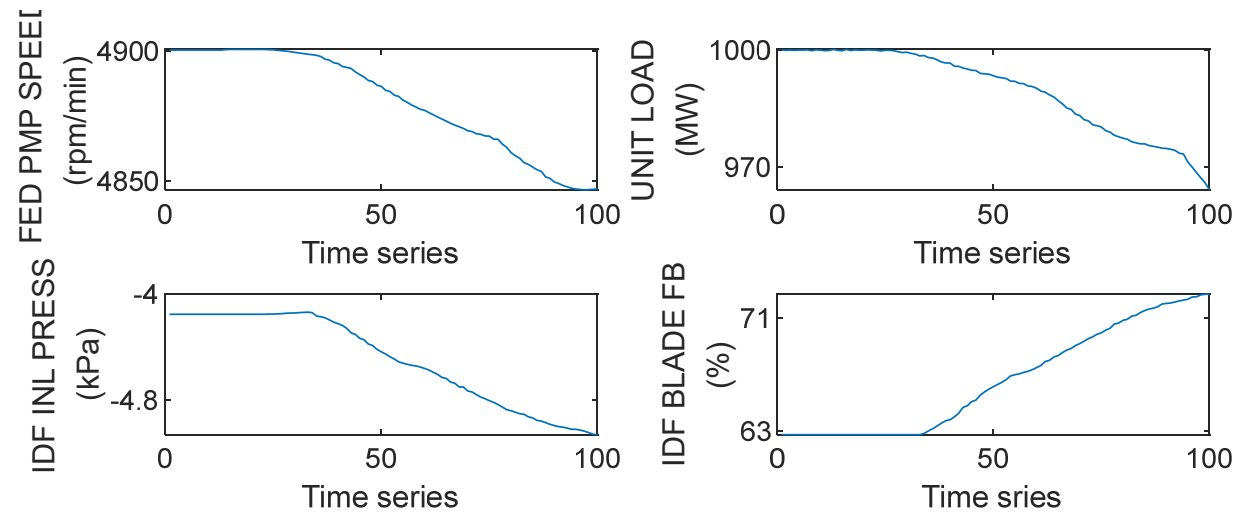

Fig1 Parameters trend under eco leakage

The setting fault is that from the $21 \mathrm{~s}$ onwards, eco leakage fault occurred at the final stage of A side is set. The figure shows the variation trend of the parameter values within 100 s before and after the fault.

As shown in Figure 1, in the early stage of the fault, especially when the leakage is small, the variation of each symptom parameter are not obvious, and it is difficult for on-site operators to notice abnormal conditions. About 58 seconds later, the DCS system releases over-temperature alarms for reheated steam temperature and overheated steam temperature. It can be seen from figure 1 that the variation trend of each parameter is clearly downward, reflecting that the unit state has occurred significantly variation.

\subsection{Leakage warning and diagnosis}

According to the fault described in the previous section, the estimated value can be obtained by establishing the parametric regression model, and the calculation formula of the state index representing the overall operating status of the unit is as follows:

$$
\mathrm{ST}=\frac{1}{1+\sqrt{\sum_{i=1}^{p}\left(x_{i}^{R}-x_{i}^{T}\right)^{2}}}
$$

In the formula, $p$ is the number of symptom parameters related to concrete faults. Obviously, a decline in the index represents a decline in unit operation state. Figure 2 shows the variation of the state index after eco leakage fault on side $\mathrm{A}$.

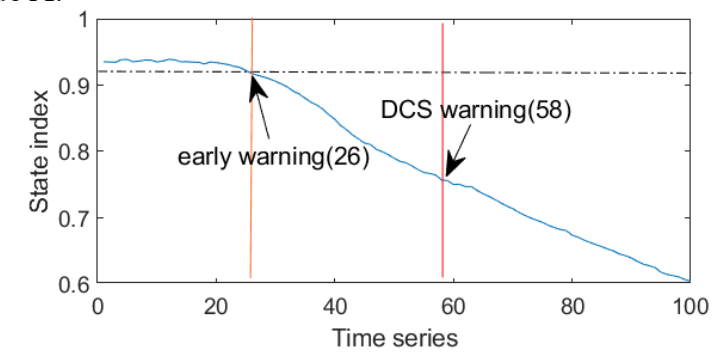

Fig2 State index trend after fault

As shown in Figure 2, the solid curve represents the variation trend of state index, and the chain line is the limit of health index warning, which is generally obtained through statistical analysis under normal working conditions. It can be seen that the state index is maintained at a relatively stable level under normal operation conditions. However, When a fault occurs, it shows a significant downward trend, reaching the warning limit at the 26th second, namely, a health warning occurs 8 seconds after the fault occurs, while the DCS gives an over-temperature alarm at about the 58th second. Obviously, the early warning signal is highly sensitive to minor degradation and has an early warning effect on faults.

When an early warning signal appears, it is necessary to further diagnose the specific fault type to provide effective information for timely processing. By using the expert knowledge base and fuzzy inference, the specific fault mode is determined. Table 3 shows the calculation results of the membership degrees of various faults after the fault occurs.

Tab.3 Membership of leakage diagnosis

\begin{tabular}{ccccc}
\hline $\begin{array}{c}\text { Time } \\
\text { point }\end{array}$ & $u_{1}$ & $u_{2}$ & $u_{3}$ & $u_{4}$ \\
\hline 19 & 0.109 & 0.09 & 0 & 0.149 \\
26 & 0.19 & 0.141 & $\mathbf{0 . 6 8 5}$ & 0 \\
30 & 0.185 & 0.141 & $\mathbf{0 . 8 5 4}$ & 0 \\
\hline
\end{tabular}

As shown in Table 3, the membership values at three time points are calculated using fuzzy inference. It can be seen that with the occurrence and deterioration of the fault, the $u_{3}$ membership has increased significantly and gradually tends to 1 . After the warning signal appears, it can be judged that the eco leakage has occurred. As time goes by, the certainty of the eco leakage becomes greater. The membership degree calculation result at the 30th second further verifies the correctness of the early warning fault identification.

\section{Conclusion}

The state parameter regression model of unit parameters is established, and the state index is constructed for the fault warning of unit faults. The fault diagnosis expert knowledge base and the fuzzy inference are used to determine the specific fault mode. A $1000 \mathrm{MW}$ unit simulation system is used to simulate the characteristics of boiler leakage fault which is used to verify the proposed method. Results show that in the early stage of fault, the method can effective warn unit failure, which verifies the 
correctness and effectiveness of the proposed method.

\section{Acknowledgement}

Supported by: Science and Technology Projects (2019) of State Grid Jiangsu Electric Power Co., Ltd. (J2019029)

\section{Reference}

1. Tan Z X, Shi C R, Huang J, et al. Statistical analysis and improvement measures of thermal power unit outage in Jiangxi power grid unified dispatching in 2019[J]. Jiangxi electric power, 2020, 40(8): 39-46.

2. Zhang Z Y, Meng Z B, Yuan H K. Zhang Zhenyu applied data mining to early warning of actual power station [J]. Power System Engineering, 2021, 37(01): 55-56.

3. Fu W A, Zhang Z F, Huang W. Research on Fault Early Warning Algorithm of Reheater in Thermal Power Plant Based on Extreme Random [J]. Journal of Shanghai University of Electric Power, 2020, 36(5): 445-450.

4. Alaei H K, Salahshoor K, Alaei H K. A new integrated on-line fuzzy clustering and segmentation methodology with adaptive PCA approach for process monitoring and fault detection and diagnosis[J]. soft computing, 2013, 17(3): 345-362.

5. Zhao H, Liu H, Hu W, et al. Anomaly detection and fault analysis of wind turbine components based on deep learning network[J]. Renewable energy, 2018, 127: 825-834.

6. Xiao L, Luo J, Ouyang C M. Research on coal mill fault prediction based on semi-supervised learning method [J]. Thermal Power Generation, 2019, 48(4): 121-127.

7. YANG $\mathrm{Hu}$, Thomas Palme, Olga Fink. Fault Detection based on Signal Reconstruction with AutoAssociative Extreme Learning Machines[J]. Engineering Application of Artificial Intelligence, 2017, 57:105-117. 